\title{
Communication
}

\section{Construction of an Artificial MicroRNA Expression Vector for Simultaneous Inhibition of Multiple Genes in Mammalian Cells}

\author{
Tao Hu ${ }^{1}$, Qiong Fu ${ }^{1}$, Ping Chen ${ }^{2}$, Li Ma ${ }^{1}$, Onsam Sin ${ }^{1}$ and Deyin Guo ${ }^{1, *}$
}

1 State Key Laboratory of Virology and The Modern Virology Research Centre, College of Life Sciences, Wuhan University, Wuhan 430072, China; E-Mails: hnhutao@sohu.com (T.H.); qiongfu@mail.utexas.edu (Q.F.); mary19840209@126.com (L.M.); soskorea@163.com (O.S.) Department of Pathophysiology, Basic Medical College, Zhengzhou University, Zhengzhou 450001, China; E-Mail: chp7779@sohu.com (P.C.)

* Author to whom correspondence should be addressed; E-Mail: dguo@whu.edu.cn; Tel. +86-27-6875-2506; Fax: +86-27-6875-2897

Received: 8 April 2009; in revised form: 16 April 2009 / Accepted: 14 May 2009 / Published: 14 May 2009

\begin{abstract}
Recently, artificial microRNA (amiRNA) has become a promising RNA interference (RNAi) technology. Here, we describe a flexible and reliable method for constructing both single- and multi-amiRNA expression vectors. Two universal primers, together with two specific primers carrying the encoding sequence of amiRNA were designed and utilized to synthesize the functional amiRNA cassette through a one-step PCR. With appropriate restriction sites, the synthesized amiRNA cassettes can be cloned into any site of different destination vectors. Using the method, we constructed both singleand multi-amiRNA expression vectors to target three reporter genes, which code firefly luciferase (Fluc), enhanced green fluorescent protein (EGFP) and $\beta$-galactosidase (LacZ), respectively. The expressions of three genes were all specifically inhibited by either the corresponding single- or the multi-amiRNA expression vector in $293 \mathrm{~T}$ cells. And the RNAi efficiency of each amiRNA produced by both single- and multi-amiRNA expression vectors was comparable.
\end{abstract}

Keywords: artificial microRNA; expression vector; multiple genes; RNA interference 


\section{Introduction}

MicroRNAs (miRNAs) are endogenously encoded $\sim 22$ nt RNAs that can cause target mRNA degradation or translation repression, and play important roles in differentiation, development, cancer, or viral infections [1]. They are most often transcribed by RNA polymerase II as long primary RNA transcripts (pri-miRNAs), which are normally capped at the $5^{\prime}$ end and polyadenylated at the 3 ' end [2,3]. Approximately 50\% of known miRNA genes are clustered [4], implying that some miRNAs are transcribed as a polycistronic pri-miRNA and processed from a single transcript [5]. The pri-miRNA is processed in the nucleus by Drosha-DGCR8 complex to liberate a 60-70 nt stem loop structure known as precursor miRNA (pre-miRNA) [6,7]. The pre-miRNA is then exported to the cytoplasm by an Exportin 5-dependent mechanism and further processed into a transient $\sim 22$ bp miRNA:miRNA* duplex (* indicates the passenger strand) by a RNase III-type enzyme Dicer [8-11]. The miRNA:miRNA* duplex is then loaded into the RNA-induced silencing complex (RISC) where the mature miRNA binds to complementary site of target mRNA to inhibit translation [12]. Recently, a significant advance in RNAi technology is the use of artificial microRNAs (amiRNAs).

The amiRNA technology employs the backbone of natural miRNAs to generate designed miRNA that can efficiently and specifically silence the genes of interest. Only the natural miRNA:miRNA* duplex sequence is replaced by the artificial one. Such a design allows amiRNAs to be processed in a similar biogenesis pathway of natural miRNA and results in functional mature amiRNAs. Zeng and colleagues first successfully utilized miR-30 backbone to produce functional amiRNAs and suppress the expression of endogenous human genes [13]. Compared with conventional siRNA and shRNA approaches, the amiRNA technology has the following advantages: 1) Easier to express multiple amiRNAs [14-16]; 2) Easier to achieve tissue-specific RNAi; 3) They confer the ability to control the timing and level of gene silencing; and 4) They provide a correlated marker to track amiRNAexpressing cells $[17,18]$.

However, the difficulty of generating amiRNA expression constructs may limit their wide application. Current methods for constructing amiRNA expression constructs require a special vector carrying the flanking sequence of natural miRNA or the synthesis of long oligonucleotides. The former, such as the pCMV-miR-30 [19], pENTR/CMV-EGFP-miR-30 [20] or SIBR [21] vectors, lack flexibility for employing various miRNA backbones, using different promoters and generating multiamiRNA expression constructs. The latter, which requires the synthesis of an approximately $96 \mathrm{nt}$ oligonucleotide template with a stem-loop structure [14,22,23], is costly and often suffers from DNA synthesis errors.

In the study, we present a rapid, flexible and reliable method for generating both single- and multiamiRNA expression vectors. This method only utilizes four short primers $(<60 \mathrm{nt})$ to synthesize the functional amiRNA cassette through a one-step PCR. The synthesized amiRNA cassette, which contain 5' cloning sites, 5' flanking region, amiRNA coding sequence, terminal loop region, amiRNA* coding sequence, $3^{\prime}$ flanking region and 3 ' cloning sites, can be cloned individually or in tandem into any destination vector with appropriate sites. With this method, we readily constructed both singleand multi-amiRNA expression vectors to inhibit the expression of EGFP, Fluc and LacZ reporter genes. Our results showed that the expression of all three reporter genes can be specifically suppressed 
by either corresponding single- or multi-amiRNA expression vector, and the RNAi efficiency of the multi-amiRNA expression vector is comparable to that of the single one.

\section{Results and Discussion}

\subsection{Construction of amiRNA expression vectors}

To validate the method, three reporter genes encoding for Fluc, EGFP and LacZ were selected as targets, and the backbone of miR-155 was employed for the expression of amiRNA. The procedure for synthesizing amiRNA cassette by a one-step PCR is depicted schematically in Figure 1a. In the first several PCR cycles, the two specific primers Fluc5 and Fluc3 were annealed through a 19 bp complimentary region of their 3' ends, and extended to yield a DNA fragment about 100 bp by KOD DNA polymerase. The overlap-extended DNA fragment was then amplified by two universal primers 155-5 and 155-3 to produce the amiRNA cassette against Fluc (amiR-Fluc) as designed. The 19-nt terminal loop region was used for annealing of two specific primers, and provided a proper annealing temperature for specific amplification. Previous research showed that during construction of miR-30based shRNA libraries [22], 97-nt DNA templates for each silencing trigger were designed to be amplified by universal primers, but only $25-60 \%$ of the clones had correct shRNA sequences. Another research also indicated that up to $50 \%$ of these 'positive' recombinant clones contained substitutions and deletions when using Taq polymerase to construct shRNA vector [24]. However, in the method provided here, all four primers for synthesis of amiRNA cassette are shorter than $60 \mathrm{nt}$ in length. The cost and possibility of errors arising from chemical synthesis of long oligonucleotides are greatly reduced. At the same time, the proofreading KOD DNA polymerase was also employed to reduce the mutation rates of $\mathrm{PCR}$ amplification.

Figure 1. The method for constructing amiRNA expression vector. (a) Schematic representation of the one-step PCR used to synthesize an amiRNA cassette. The black rectangle represents amiRNA. (b) Results of the one-step PCR. The 180 nt DNA fragements are indicated by arrowhead. Lane M, 100 bp DNA ladder; lane 1, amiR-Fluc; lane 2, amiR-EGFP; lane 3, amiR-LacZ. (c) Strategy for generating the multi-amiRNA expression vector.

a

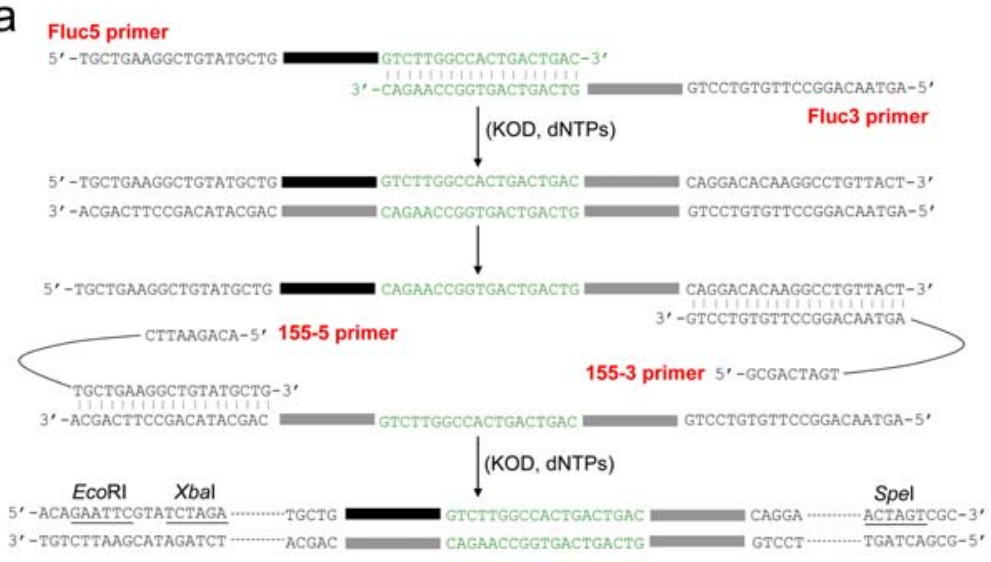

b

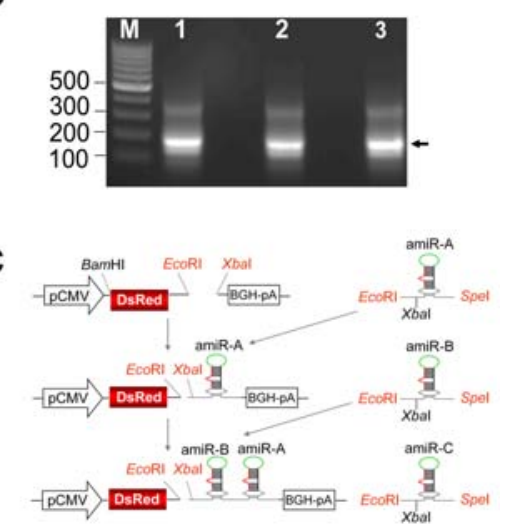


The resulting PCR products were analyzed on 1.2\% agarose gel. Figure $1 \mathrm{~b}$ shows that the synthesized amiRNA cassettes have an expected molecular size of about $180 \mathrm{bp}$. These PCR products were then recovered by the glass beads method, which provides high extraction efficiency for small DNA fragments. Subsequently, amiRNA cassettes against Fluc, EGFP (amiR-EGFP) or LacZ (amiRLacZ) were inserted individually or in tandem into the pDsRed vector via unique but compatible restriction sites (Figure 1c). The resulting vectors were named pDsRed-amiR-Fluc, pDsRed-amiREGFP, pDsRed-amiR-LacZ, and pDsRed-amiR-ZGF (contains the cassettes of amiR-LacZ, amiR-EGFP and amiR-Fluc in tandem), respectively. In these vectors, the red fluorescence gene (DsRed) tightly linked to the amiRNA cassette allows us to track the efficiency of transfection. All amiRNA expression vectors were verified by sequencing with BGH reverse primer. Using this method, we obtained at least two correct constructs out of three for each amiRNA expression vector.

\subsection{Suppression of firefly luciferase expression by amiR-Fluc expression vectors}

To test whether these amiRNA expression vectors would be functionally effective, RNAi efficiencies against corresponding target genes were evaluated by transient transfection assays. Firefly luciferase expression vector pGL3-CMV was co-transfected with amiR-Fluc expression vector pDsRed-amiR-Fluc or pDsRed-amiR-ZGF into $293 \mathrm{~T}$ cells.

Figure 2. Suppression of firefly luciferase expression by either single amiRNA expression vector pDsRed-amiR-Fluc or multi-amiRNA expression vector pDsRed-amiR-ZGF in $293 \mathrm{~T}$ cells. (a) The structure and sequence of pri-amiR-Fluc. Green letters represent the terminal loop region; red letters represent the amiR-Fluc sequence; gray letters represent the flanking sequence. (b) Luciferase expression vector pGL-CMV (50 ng) was cotransfected into $293 \mathrm{~T}$ cells with $150 \mathrm{ng}$ amiR-Fluc expression vectors or pDsRed-amiREGFP. Firefly luciferase activities were measured 24 hours after transfection. The luciferase activity in the presence of pDsRed-amiR-EGFP was set at 100\%. Error bars represent the standard deviation from three independent experiments $(p<0.001$ for both amiR-Fluc expression vectors when compared to the control vector). (c) Detection of amiR-Fluc expression by Northern blotting. The amiRNAs migrated slightly slower than DNA markers and at an approximate size of $28 \mathrm{nt}$ DNA oligonucleotide. The 28S rRNA served as an internal control for equal RNA loading.

a

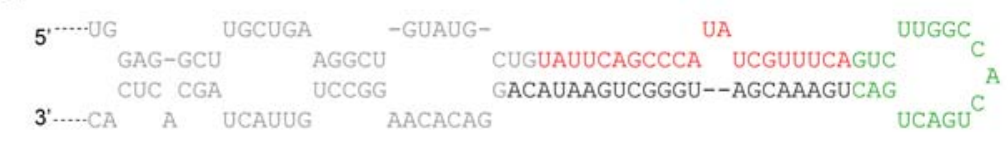

b

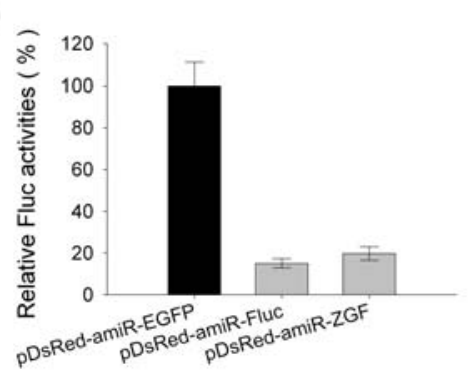

C

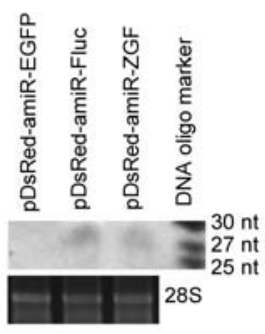


The structure and sequence of pri-amiR-Fluc is shown in Figure 2a. About $24 \mathrm{~h}$ after transfection, the luciferase activity was measured. The activity in control cells co-transfected with pGL3-CMV and pDsRed-miR-EGFP was set as $100 \%$. Figure $2 \mathrm{~b}$ shows that pDsRed-amiR-Fluc and pDsRed-amiRZGF all significantly reduced luciferase activity. The Northern blot analyses using a probe complementary to amiR-Fluc sequence also confirmed that both pDsRed-amiR-Fluc and pDsRedamiR-ZGF produced the mature amiR-Fluc of about $22 \mathrm{nt}$ in transfected cells (Figure 2c).

\subsection{Efficient suppression of EGFP expression by amiR-EGFP expression vectors}

We next examined the inhibition effect on EGFP expression induced by amiR-EGFP expression vectors. The pDsRed-amiR-EGFP or pDsRed-amiR-ZGF was co-transfected with pEGFP-C1 vector into $293 \mathrm{~T}$ cells, and the RNAi effect was evaluated by fluorescent microscopy and Western blotting.

Figure 3. Efficient suppression of EGFP expression by pDsRed-amiR-EGFP or pDsRedamiR-ZGF in $293 \mathrm{~T}$ cells. (a) EGFP expression vector pEGFP-C1 (100 ng) was co-transfected with $100 \mathrm{ng}$ amiR-EGFP expression vectors or pDsRed-amiR-Fluc into $293 \mathrm{~T}$ cells. EGFP expression was detected by fluorescence microscopy. (b) EGFP expression was analyzed by Western blotting. The Western blotting shows a representative result from three independent experiments. (c) After normalizing to $\beta$-actin, the inhibition level induced by pDsRed-amiR-EGFP or pDsRed-amiR-ZGF was assessed by densitometry. Normalized values (mean \pm SD) from three independent Western blotting experiments are shown $(p<0.001$ for both amiR-EGFP expression vectors when compared to the control vector).

a

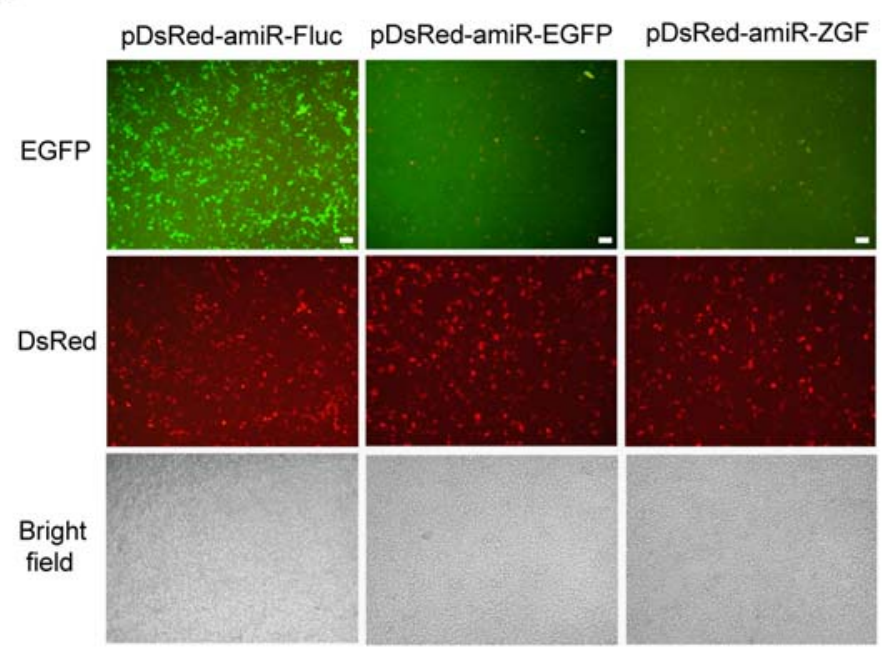

b

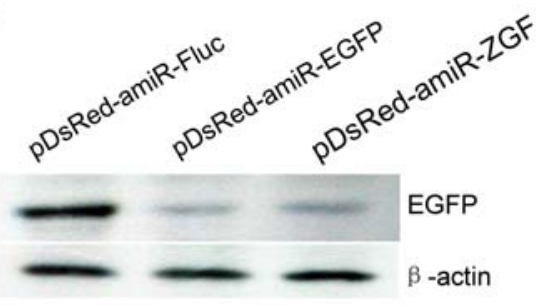

C

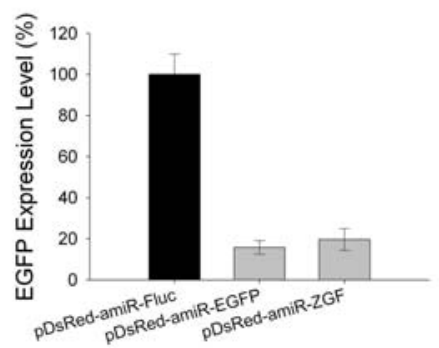

As shown in Figure 3a, the expression of EGFP was obviously suppressed by either pDsRed-amiREGFP or pDsRed-amiR-ZGF. The observation under fluorescent microscope was further confirmed by the Western blotting result (Figure 3b). After normalizing to $\beta$-actin, EGFP expression level in the pDsRed-amiR-EGFP- or pDsRed-amiR-ZGF-transfected $293 \mathrm{~T}$ cells was only $16 \%$ or $19 \%$ of the control (pDsRed-amiR-Fluc-transfected) cells, as determined by densitometry (Figure 3c). At the same time, the results of red fluorescence observations suggested that the marker protein DsRed were 
expressed at reasonable levels in all cells transfected with amiRNA expression vectors (Figure 3a). Such arrangement was also proved to be effective by other reports. However, when the ORF of DsRed was placed at the 3' end of amiRNA cassette, the DsRed was poorly expressed (data not shown). Thus, placing the reporter gene at $5^{\prime}$ end of amiRNA cassette would be a better approach to co-express amiRNA along with the marker protein. In addition, a strategy to express amiRNA from an intron was also used to improve the marker gene expression $[17,18]$.

\subsection{Efficient inhibition of $\beta$-galactosidase expression by amiR-LacZ expression vectors}

The expression of LacZ reporter gene was also specifically suppressed by either pDsRed-amiRLacZ or pDsRed-amiR-ZGF in 293T cells (Figure 4a), and their RNAi efficiencies were 79\% and 74\%, respectively (Figure 4b). Taken together, all amiRNA expression vectors constructed in the study were functionally effective, and the expressions of all three reporter genes were specifically suppressed by either corresponding single- or multi-amiRNA expression vector. These results shows that multiple amiRNAs can be co-expressed from a single transcript, and RNAi efficiency of each is comparable to that produced by the single amiRNA expression vector.

Figure 4. Inhibition of $\beta$-galactosidase expression by pDsRed-amiR-LacZ or pDsRedamiR-ZGF in 293T cells. (a) The pSV- $\beta$-Galactosidase expression vector (100 ng) was cotransfected with $100 \mathrm{ng}$ amiR-LacZ expression vectors or pDsRed-amiR-EGFP into 293T cells. The $\beta$-galactosidase expression was detected by X-gal staining. (b) $\beta$-Galactosidase activity was further determined by the Beta-Glo assay system kit (Promega) according to the manufacturer's instructions. A background value was subtracted from each reading before comparison. The $\beta$-galactosidase activity in the presence of pDsRed-amiR-EGFP was set at $100 \%$. Error bars represent standard deviation from three independent experiments $(p<0.001$ for both amiR-LacZ expression vectors when compared to the control vector).
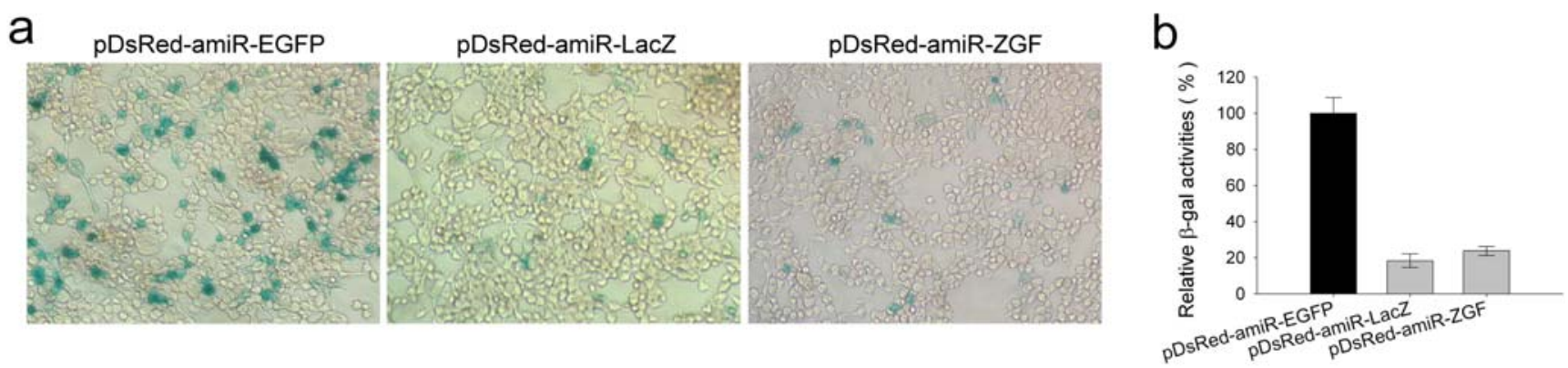

Endogenous pri-miRNAs share some similar structural characteristics that comprise a stem, a terminal loop, long flanking sequences, and some internal loops or bulges, which likely contribute to an efficient processing in miRNA pathway $[25,26]$. The sequence flanking pre-miRNA has been proven critical for pri-miRNA to be processed into pre-miRNA by Drosha-DGCR8 complex [27,28]. As reported for miR-223, $40 \mathrm{nt}$ of flanking sequence on each side of pre-miR-223 are necessary for the maturation of miR-223 [29]. Chung et al. delimited a smallest fully functional fragment of $108 \mathrm{nt}$ (BIC 134-241) that is sufficient to produce mature miR-155 in mammalian cells [21]. The miR-30 can be 
generated correctly and efficiently when the pre-miRNA is flanked by $22 \mathrm{nt}$ flanking sequences at its 5' side and $15 \mathrm{nt}$ at its 3' side [18]. Sun et al. also used an extended miR-30 hairpin of $118 \mathrm{nt}$ as a backbone for amiRNA expression cassette [14]. Based on these previous results, we reasoned that $40 \mathrm{nt}$ flanking sequence on each side of pre-miRNA are essential and sufficient for the processing and maturation of most miRNAs. Therefore, a functional amiRNA cassette should usually contain 5' flanking region of at least $40 \mathrm{nt}$ from natural pri-miRNA, encoding sequence of amiRNA, the terminal loop region, coding sequence of amiRNA* and 3' flanking region of at least $40 \mathrm{nt}$ from natural primiRNA, in the order of a 5' to $3^{\prime}$ direction. Appropriate restriction sites can be introduced at both ends of cassette. This might be considered as a general rule for designing the amiRNA or even natural miRNA cassette. According to this rule, a functional amiRNA cassette plus cloning sites would be approximately $170 \mathrm{bp}$ in length. With appropriate sites, the synthesized amiRNA cassette can be inserted into any site of different expression vectors, which would greatly enhance the flexibility to construct amiRNA expression vectors.

Many endogenous miRNA genes are found in close proximity to others. These natural miRNAs can be generated from a single polycistronic primary transcript [30], and several groups have demonstrated that some artificial miRNAs could be expressed from one single RNA transcript. It's has been observed that multiple copies of an amiRNA expressed from a single polycistronic transcript could enhance the RNAi effect [16,21]. But surprisingly, it has also been reported that miRNA yields and gene silencing effects were substantially increased when two distinct amiRNAs co-expressed in tandem from a single transcript [14]. In our study, the expression of all three reporter genes can be suppressed at a high level by either corresponding single- or multi-amiRNA expression vector, and the silencing effects are comparable between the single and multiple one. Therefore, by using amiRNA technology, RNA silencing of multiple genes can be readily and simultaneously achieved.

\section{Experimental Section}

\subsection{Synthesis of amiRNA cassette by one-step PCR}

Three reporter genes coding for Fluc, EGFP and LacZ were selected to test the amiRNA-mediated RNAi efficiency. The amiR-Fluc was designed according to a previous report [17]. The amiR-EGFP and amiR-LacZ were designed using the Block-it RNAi designer (http://rnaidesigner.invitrogen.com). The amiRNAs target sequences are as follows: coding regions $200-220$ (5'TGAAACGATATGGGCTGAATA-3') for Fluc; 123 - 143 (5'-CAAGCTGACCCTGAAGTTCAT-3') for EGFP; 642 - 662 (5'-GACTACACAAATCAGCGATTT-3') for LacZ. Two universal primers and three pairs of specific primers were designed and then synthesized by Invitrogen. The universal primer 155-5 includes sequence of 5'-flanking region of pre-miR-155, EcoRI and $X b a \mathrm{I}$ restriction sites. The universal primer 155-3 includes reverse complement sequence of 3'-flanking region of pre-miR-155 and SpeI site. The specific primer pairs Fluc1/Fluc2, EGFP1/EGFP2 and LacZ1/LacZ2 carry the coding sequence of amiRNA against the corresponding target gene and have a $19 \mathrm{nt}$ complementary overlap at their 3' ends. Sequences of all primers are listed in Table 1. The two universal primers and one pair of specific primers were utilized to synthesize an amiRNA cassette by one-step PCR. Schematic representation of one-step PCR is shown in Figure 1a. 
PCR amplification was performed in a $50 \mu \mathrm{L}$ volume containing 1 x PCR reaction buffer, 25 pmol each of universal primers, 5 pmol of each specific primer, $1 \mathrm{mM} \mathrm{MgSO}_{4}, 0.2 \mathrm{mM}$ dNTPs and 1 unit KOD-Plus DNA polymerase (TOYOBO). The PCR program was as follows: $94{ }^{\circ} \mathrm{C}$ for 3 min, 35 cycles of $15 \mathrm{sec}$ at $94{ }^{\circ} \mathrm{C}, 30 \mathrm{sec}$ at $55^{\circ} \mathrm{C}, 15 \mathrm{sec}$ at $68^{\circ} \mathrm{C}$ and a final extension for $8 \mathrm{~min}$ at $68{ }^{\circ} \mathrm{C}$. The PCR products were separated on a $1.2 \%$ agarose gel stained with ethidium bromide and compared to 100 bp DNA ladder (Fermentas).

Table 1. Oligonucleotides used in this paper.

\begin{tabular}{ll}
\hline Name & Sequence (5’-3’) \\
\hline $155-5$ & ACAGAATTCGACTCTAGAATCCTCTGGCTGCTGGAGGCTTGCTGAAGGCTGTATGCTG \\
$155-3$ & GCGACTAGTACGGTGGCCATTTGTTCCATGTGAGTGCTAGTAACAGGCCTTGTGTCCTG \\
Fluc5 & TGCTGAAGGCTGTATGCTGTATTCAGCCCATATCGTTTCAGTCTTGGCCACTGACTGAC \\
Fluc3 & AGTAACAGGCCTTGTGTCCTGTATTCAGCCCATCGTTTCAGTCAGTCAGTGGCCAAGAC \\
EGFP5 & TGCTGAAGGCTGTATGCTGATGAACTTCAGGGTCAGCTTGGTCTTGGCCACTGACTGAC \\
EGFP3 & AGTAACAGGCCTTGTGTCCTGATGAACTTCAGTCAGCTTGGTCAGTCAGTGGCCAAGAC \\
LacZ5 & TGCTGAAGGCTGTATGCTGAAATCGCTGATTTGTGTAGTCGTCTTGGCCACTGACTGAC \\
LacZ3 & AGTAACAGGCCTTGTGTCCTGAAATCGCTGATGTGTAGTCGTCAGTCAGTGGCCAAGAC \\
\hline & $*$ The underlined letters present the sequences of amiRNAs.
\end{tabular}

\subsection{Plasmid construction}

The coding sequence of red fluorescent protein (DsRed) was cleaved from pDsRed2 vector (Clontech) with BamHI and EcoRI, and then inserted into pVAX1 vector (Invitrogen) to generate vector pDsRed. The synthesized amiR-Fluc, amiR-EGFP or amiR-LacZ cassette was digested with EcoRI and SpeI, and then cloned into the pDsRed vector, which was digested with EcoRI and $X b a \mathrm{I}$ (compatible end with SpeI), to generate pDsRed-amiRFluc, pDsRed-amiREGFP and pDsRedamiRLacZ vectors. The strategy for generating multi-amiRNA expression vector is illustrated in Figure 1c. These constructs can be identified by sequencing with BGH reverse primer.

\subsection{Cell culture and DNA transfection}

HEK 293 T cells were plated in 96-well plates at $1.5 \times 10^{4}$ cells per well and grown as a monolayer in DMEM (Invitrogen) supplemented with $10 \%$ fetal calf serum at $37{ }^{\circ} \mathrm{C}$ and $5 \% \mathrm{CO}_{2}$. Cells at $70 \%$ confluence were co-transfected with the indicated amounts of plasmids using Lipofectamine 2,000 reagent (Invitrogen) according to the manufacturer's instructions.

\subsection{Reporter assays}

Firefly luciferase activity assay was performed 24 hours after transfection using a Steady-Glo Luciferase Assay System Kit (Promega), and chemiluminescence was measured in a luminometer (Tecan). The ß-galactosidase activity was quantified by using the Beta-Glo Assay System (Promega) 
in accordance with the manufacturer's protocol. The X-gal staining of cells was performed as described previously [31].

\subsection{Western blotting}

Western blotting was performed as described previously [32]. EGFP expression was detected by using mouse monoclonal anti-GFP antibody (Lab Vision) as primary antibodies, and probed with a goat anti-mouse IgG horseradish peroxidase conjugate (Pierce).

\subsection{Northern blotting}

Twenty-four hours after transfection, total RNA was extracted using TRIzol reagent (Invitrogen) following the manufacturer's protocol. Thirty micrograms of total RNA was separated by electrophoresis on a $15 \%$ denaturing polyacrylamide gel, and blotted onto Hybond $\mathrm{N}^{+}$membranes. The 25-nt, 27-nt and 30-nt DNA oligonucletides were also loaded on the same gel to provide molecular weight markers. RNA was immobilized by UV crosslinking. Hybridization was carried out at $37{ }^{\circ} \mathrm{C}$ using Hybridization Buffer (Innogent). The amiR-Fluc was probed with a digoxigenin-labeled 21-nt DNA (5'- TGAAACGATATGGGCTGAATA -3') using DIG nucleic acid detection kit II (Innogent).

\section{Conclusions}

In summary, we propose a general rule for amiRNA design. According to this rule, a one-step PCRbased method was developed to construct both single- and multi-amiRNA expression vectors. All amiRNA expression vectors constructed in this study could specifically inhibit the expression of corresponding target genes in mammalian cells. The RNAi efficiency of each amiRNA produced by the multi-amiRNA vector is comparable to that of the single one. With the method, we constructed more than 100 amiR-Luc expression vectors using different expression patterns or with different primiRNA structures to further optimize this amiRNA expression system. It should also be mentioned that this method can be extended to employ other miRNA backbones. In our lab, the backbones of miR-16, miR-206, and miR-331 were all successfully employed to express functional amiRNAs (unpublished data). These results show that our method for constructing amiRNA expression vector is highly flexible and reliable and will facilitate the application of amiRNA technology in basic research and gene therapy. In addition, the multi-amiRNA expression vector also make it more convenient and efficient to increase the silencing efficiency of a target gene or a virus, to knockdown the expression of multiple related genes, or to inhibit the replication of two or more viruses simultaneously.

\section{Acknowledgements}

This study was supported by China "863" program (grant 2006AA02Z123) and the MOE "111" project \#B06018. 


\section{References}

1. Bushati, N.; Cohen, S.M. MicroRNA functions. Annu. Rev. Cell Dev. Biol. 2007, 23, 175-205.

2. Lee, Y.; Kim, M.; Han, J.; Yeom, K.H.; Lee, S.; Baek, S.H.; Kim, V.N. MicroRNA genes are transcribed by RNA polymerase II. EMBO J. 2004, 23, 4051-4060.

3. Cai, X.; Hagedorn, C.H.; Cullen, B.R., Human microRNAs are processed from capped, polyadenylated transcripts that can also function as mRNAs. RNA 2004, 10, 1957-1966.

4. Kim, V.N.; Nam, J.W. Genomics of microRNA. Trends Genet. 2006, 22, 165-173.

5. Suh, M.R.; Lee, Y.; Kim, J.Y.; Kim, S.K.; Moon, S.H.; Lee, J.Y.; Cha, K.Y.; Chung, H.M.; Yoon, H.S.; Moon, S.Y.; Kim, V.N.; Kim, K.S. Human embryonic stem cells express a unique set of microRNAs. Dev. Biol. 2004, 270, 488-498.

6. Lee, Y.; Ahn, C.; Han, J.; Choi, H.; Kim, J.; Yim, J.; Lee, J.; Provost, P.; Radmark, O.; Kim, S.; Kim, V.N. The nuclear RNase III Drosha initiates microRNA processing. Nature 2003, 425, 415-419.

7. Han, J.; Lee, Y.; Yeom, K.H.; Kim, Y.K.; Jin, H.; Kim, V.N., The Drosha-DGCR8 complex in primary microRNA processing. Genes Dev. 2004, 18, 3016-3027.

8. Lund, E.; Guttinger, S.; Calado, A.; Dahlberg, J.E.; Kutay, U. Nuclear export of microRNA precursors. Science 2004, 303, 95-98.

9. Yi, R.; Qin, Y.; Macara, I.G.; Cullen, B.R. Exportin-5 mediates the nuclear export of premicroRNAs and short hairpin RNAs. Genes Dev. 2003, 17, 3011-3016.

10. Hutvagner, G.; McLachlan, J.; Pasquinelli, A.E.; Balint, E.; Tuschl, T.; Zamore, P.D. A cellular function for the RNA-interference enzyme Dicer in the maturation of the let-7 small temporal RNA. Science 2001, 293, 834-838.

11. Ketting, R.F.; Fischer, S.E.; Bernstein, E.; Sijen, T.; Hannon, G.J.; Plasterk, R.H. Dicer functions in RNA interference and in synthesis of small RNA involved in developmental timing in C. elegans. Genes Dev. 2001, 15, 2654-2659.

12. Gregory, R.I.; Chendrimada, T.P.; Cooch, N.; Shiekhattar, R. Human RISC couples microRNA biogenesis and posttranscriptional gene silencing. Cell 2005, 123, 631-640.

13. Zeng, Y.; Wagner, E.J.; Cullen, B.R. Both natural and designed micro RNAs can inhibit the expression of cognate mRNAs when expressed in human cells. Mol. Cell 2002, 9, 1327-1333.

14. Sun, D.; Melegari, M.; Sridhar, S.; Rogler, C.E.; Zhu, L. Multi-miRNA hairpin method that improves gene knockdown efficiency and provides linked multi-gene knockdown. Biotechniques 2006, 41, 59-63.

15. Zhu, X.; Santat, L.A.; Chang, M.S.; Liu, J.; Zavzavadjian, J.R.; Wall, E.A.; Kivork, C.; Simon, M.I.; Fraser, I.D. A versatile approach to multiple gene RNA interference using microRNA-based short hairpin RNAs. BMC Mol. Biol. 2007, 8, 98.

16. Zhou, H.; Huang, C.; Xia, X.G. A tightly regulated Pol III promoter for synthesis of miRNA genes in tandem. Biochim. Biophys. Acta 2008, 1779, 773-779.

17. Du, G.; Yonekubo, J.; Zeng, Y.; Osisami, M.; Frohman, M.A. Design of expression vectors for RNA interference based on miRNAs and RNA splicing. FEBS J. 2006, 273, 5421-5427.

18. Zhou, H.; Xia, X.G.; Xu, Z. An RNA polymerase II construct synthesizes short-hairpin RNA with a quantitative indicator and mediates highly efficient RNAi. Nucleic Acids Res. 2005, 33, e62. 
19. Zeng, Y.; Cai, X.; Cullen, B.R. Use of RNA polymerase II to transcribe artificial microRNAs. Methods Enzymol. 2005, 392, 371-380.

20. Gou, D.; Zhang, H.; Baviskar, P.S.; Liu, L. Primer extension-based method for the generation of a siRNA/miRNA expression vector. Physiol. Genomics 2007, 31, 554-562.

21. Chung, K.H.; Hart, C.C.; Al-Bassam, S.; Avery, A.; Taylor, J.; Patel, P.D.; Vojtek, A.B.; Turner, D.L. Polycistronic RNA polymerase II expression vectors for RNA interference based on BIC/miR-155. Nucleic Acids Res. 2006, 34, e53.

22. Chang, K.; Elledge, S.J.; Hannon, G.J. Lessons from Nature: microRNA-based shRNA libraries. Nat. Methods 2006, 3, 707-714.

23. Paddison, P.J.; Cleary, M.; Silva, J.M.; Chang, K.; Sheth, N.; Sachidanandam, R.; Hannon, G.J. Cloning of short hairpin RNAs for gene knockdown in mammalian cells. Nat. Methods 2004, 1 , 163-167.

24. McIntyre, G.J.; Fanning, G.C. Design and cloning strategies for constructing shRNA expression vectors. BMC Biotechnol. 2006, 6, 1.

25. Saetrom, P.; Snove, O.; Nedland, M.; Grunfeld, T.B.; Lin, Y.; Bass, M.B.; Canon, J.R. Conserved microRNA characteristics in mammals. Oligonucleotides 2006, 16, 115-144.

26. Ritchie, W.; Legendre, M.; Gautheret, D. RNA stem-loops: to be or not to be cleaved by RNAse III. RNA 2007, 13, 457-462.

27. Zeng, Y.; Cullen, B.R. Efficient processing of primary microRNA hairpins by Drosha requires flanking nonstructured RNA sequences. J. Biol. Chem. 2005, 280, 27595-27603.

28. Han, J.; Lee, Y.; Yeom, K.H.; Nam, J.W.; Heo, I.; Rhee, J.K.; Sohn, S.Y.; Cho, Y.; Zhang, B.T.; Kim, V.N. Molecular basis for the recognition of primary microRNAs by the Drosha-DGCR8 complex. Cell 2006, 125, 887-901.

29. Chen, C.Z.; Li, L.; Lodish, H.F.; Bartel, D.P. MicroRNAs modulate hematopoietic lineage differentiation. Science 2004, 303, 83-86.

30. Cullen, B.R. Transcription and processing of human microRNA precursors. Mol. Cell 2004, 16, 861-865.

31. Li, Z.; Xiong, Y.; Peng, Y.; Pan, J.; Chen, Y.; Wu, X.; Hussain, S.; Tien, P.; Guo, D. Specific inhibition of HIV-1 replication by short hairpin RNAs targeting human cyclin T1 without inducing apoptosis. FEBS Lett. 2005, 579, 3100-3106.

32. Hu, T.; Fu, Q.; Chen, P.; Zhang, K.; Guo, D. Generation of a stable mammalian cell line for simultaneous expression of multiple genes by using $2 \mathrm{~A}$ peptide-based lentiviral vector. Biotechnol. Lett. 2009, 31, 353-359.

(C) 2009 by the authors; licensee Molecular Diversity Preservation International, Basel, Switzerland. This article is an open-access article distributed under the terms and conditions of the Creative Commons Attribution license (http://creativecommons.org/licenses/by/3.0/). 